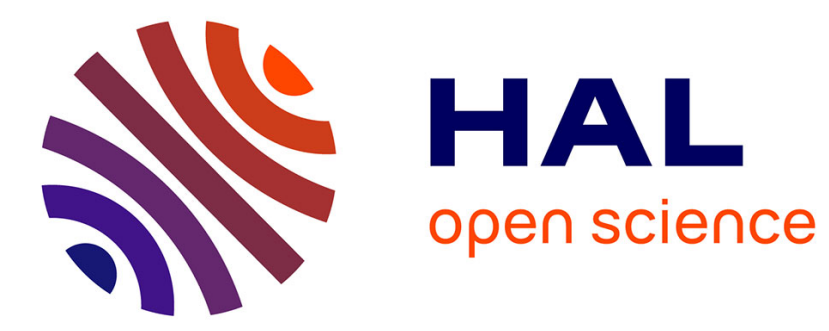

\title{
Une préhampe magdalénienne en bois de renne aux Petits Guinards (Allier, France)
}

François-Xavier Chauvière, Laure Fontana, Laurent Lang, Georges Bonani, Irka Hajdas

\section{- To cite this version:}

François-Xavier Chauvière, Laure Fontana, Laurent Lang, Georges Bonani, Irka Hajdas. Une préhampe magdalénienne en bois de renne aux Petits Guinards (Allier, France). Comptes Rendus. Palevol, 2006, 5, pp.725-733. halshs-00085582

\section{HAL Id: halshs-00085582 https://shs.hal.science/halshs-00085582}

Submitted on 11 Jun 2020

HAL is a multi-disciplinary open access archive for the deposit and dissemination of scientific research documents, whether they are published or not. The documents may come from teaching and research institutions in France or abroad, or from public or private research centers.
L'archive ouverte pluridisciplinaire HAL, est destinée au dépôt et à la diffusion de documents scientifiques de niveau recherche, publiés ou non, émanant des établissements d'enseignement et de recherche français ou étrangers, des laboratoires publics ou privés. 


\title{
Paléontologie humaine et préhistoire / Human paleontology and prehistory
}

\section{Une préhampe magdalénienne en bois de renne aux Petits Guinards (Allier, France)}

\author{
François-Xavier Chauvière ${ }^{\mathrm{a}, *}$, Laure Fontana ${ }^{\mathrm{b}}$, Laurent Lang ${ }^{\mathrm{c}}$, \\ Georges Bonani ${ }^{\mathrm{d}}$, Irka Hajdas ${ }^{\mathrm{e}}$ \\ a Institut de préhistoire de l'université de Neuchâtel, Laténium, \\ parc et musée d'archéologie de Neuchâtel, espace Paul-Vouga, CH-2068 Hauterive, Suisse \\ b "Économies, Sociétés, Environnements préhistoriques », CNRS, UMR 6636, maison méditerranéenne des sciences de l'Homme, \\ 5, rue du Château-de-l'Horloge, BP 647, 13094 Aix-en-Provence cedex 02, France \\ c Inrap centre, 15 rue du Commerce, 36600 Lye, France \\ d Institute for particle physics, ETH-Hoenggerberg, HPK H3O, CH-8093 Zurich, Suisse \\ ${ }^{\mathrm{e}}$ AMS ${ }^{14} \mathrm{C}$ Lab, ETH Hoenggerberg, HPK H27, CH-8093 Zurich, Suisse
}

Reçu le 9 septembre 2005 ; accepté après révision le 5 décembre 2005

Disponible sur internet le 20 mars 2006

Présenté par Yves Coppens

\begin{abstract}
Résumé
Le gisement archéologique des Petits Guinards, à Creuzier-le-Vieux (Allier, France), a livré une série importante, à l'échelle du Massif central, d'objets travaillés en matières dures d'origine animale. Au sein des différentes catégories techniques et fonctionnelles représentées, un objet en bois de renne est interprété comme une préhampe de projectile. Cette pièce exceptionnelle fut fabriquée hors du gisement où elle a été abandonnée à l'état de fragments. Sa datation par SMA ${ }^{14} \mathrm{C}$ confirme la présence de groupes humains au tout début du Dryas ancien dans ce secteur septentrional du Massif central. Pour citer cet article : F.-X. Chauvière et al., C. R. Palevol 5 (2006). (C)
\end{abstract}

\section{Abstract}

A reindeer antler foreshaft from the Magdalenian site of the 'Petits Guinards' (Allier, France). The site of the 'Petits Guinards' in Creuzier-le-Vieux (Allier, France) yielded an important assemblage, at the scale of the Massif central, of bone industry. Among the different technical and functional categories represented, a fragment of reindeer antler is interpreted as the foreshaft of a projectile. This exceptional artefact was made out of the site where it had been left. The radiocarbon date of this foreshaft confirms the presence of human groups in the north of Massif central at the start of the Lower Dryas. To cite this article: F.-X. Chauvière et al., C. R. Palevol 5 (2006).

(C)

Mots clés : Bois de renne ; Datation SMA ${ }^{14} \mathrm{C}$; Industrie osseuse ; Magdalénien ; Massif central ; Préhampe ; Projectiles ; France

Keywords: Foreshaft; Magdalenian; Massif central; Projectiles; Radiocarbon dates; Reindeer antler; Worked bone industry; France

\footnotetext{
* Auteur correspondant.

Adresses e-mail : francois-xavier.chauviere@unine.ch (F.-X. Chauvière), lfontana@mmsh.univ-aix.fr (L. Fontana), fionalaurent@wanadoo.fr (L. Lang), bonani@phys.ethz.ch (G. Bonani), hajdas@phys.ethz.ch (I. Hajdas).1
} 


\section{Abridged English version}

\section{Introduction}

The 'Petits Guinards' (Creuzier-le-Vieux, Allier), discovered in 1981, was a rockshelter, located along the Allier River. Two recent seasons of excavation yielded about 125000 archaeological remains (lithic and bone industries, faunal remains). Preliminary investigations revealed representative assemblages $[12,13]$. The various studies carried out about this site are part of an interdisciplinary research project focusing on the Upper Palaeolithic of the Massif Central region.

The amount of reindeer antler remains (900) and the 74 pieces of bone industry (bone, ivory, cervid antler, tooth, and shell) are a distinctive feature of this site: such bone industry is very rare at the regional scale [6]. The study of this assemblage was immediately submitted to a zooarchaeological analysis. Among this bone industry, we found an exceptional worked bone: a fragment of reindeer antler, which, according to us, could be a foreshaft. Such an artefact is the middle component of a composite weapon, which is positioned between the projectile shaft and its extremity (i.e., the point). It was dated as part of a direct dating program of archaeological bone industry (Zurich Institute for Particle Physics). We present here this fragment of a foreshaft, broken in five pieces and incomplete.

\section{Raw material, technology, and functional hypothesis}

This item was made in a very large reindeer antler, which comes from an adult male, as the compact bone tissue thickness $(10 \mathrm{~mm})$ confirms it (Fig. 1). Straight and regular over the whole length $(290 \mathrm{~mm})$, it was extracted from the antler beam. A break 'en languette' is rounded over almost the whole length.

It gets gradually thinner and flat toward its narrowest extremity, which shows a double bevel with an oval section and is striated on one of its two sides. This bevel's shape indicates that the piece was intended to be stuck into a handle. This is confirmed by the rough appearance of the ridges on one side destined to the sticking on to another component easy [1]. The break 'en languette' leads us to think that there was no ligature at this place $[1,24]$.

On its broken part, two axial grooves had been made in the bone tissues. The extraction was done by obtaining a double grooved surface on the length; then the artefact was scraped and polished. These grooves, whose original length is unknown, were made in order to fit other essential components. We can partially see such a groove on another worked bone from Les Petits Guinards: a decorated chisel, made from a rensed worked bone of the same size, could be interpreted as the same object as the one we are describing here (Fig. 2).

It is clear that this item is a component of a composite piece stuck in a handle, probably on a wooden shaft.

The rectangular section does not seem to fit for a use as a projectile point/tip and the broken part (on the opposite end) was certainly not a point. In the same way, it can't be the distal end of a spear thrower, stuck on a handle made of perishable material (e.g., wood) and whose hook would be added [20].

What would such grooves, whose width and depth measurements are much higher than for most grooved projectile heads documented, be aimed at? [18]. It is quite difficult to argue that lithic microliths, such as backed bladelets, were fixed with an adhesive inside the grooves of this foreshaft. First, if we compare the general size of backed bladelets from the 'Petits Guinards' to the width and depth of the grooves, the latter are too wide and too thick: the cutting edge of most of lithic artefacts does not really stick out of the groove and therefore would not be particularly efficient. Second, the only backed tools that could be inserted in the grooves seem to come from a more recent context (Epipaleolithic?) than the one of the foreshaft.

The morphology of this artefact and the fact that the backed bladelets do not fit in the grooves lead us to propose that it is a foreshaft, as we already know some examples in the Upper Palaeolithic [4,20-22]. Indeed, it is highly likely that these grooves were used to insert either a point or another intermediate component. The contextual analysis of the whole bone industry allows us to argue that bone or wooden points, similar to those discovered at the site, fit the foreshaft. These points - or rather bi-points, straight with a triangular section could have worn or not barb rows made of lithic blanks.

The setting could be supported by an adhesive or a ligature (Fig. 3), which was not set at the actual distal end but lower on the foreshaft (i.e., fracture 'en languette'). Finally, the considerable weight of such a setting would be more fitting for a spear thrower or a thrusting weapon than for a bow [3].

This foreshaft is an exceptional weapon which is only known in seven French sites (i.e., Le Colombier, le Tuc d'Audoubert, Castanet, Longueroche, La Madeleine, Isturitz, Fontalès) [4] and its discovery (as two fractured pieces, see above), within an area where bone industry is very rare, raises some questions. 


\section{Radiocarbon dates of the foreshaft: implications}

We got an AMS ${ }^{14} \mathrm{C}$ date (ETH-28777, $14970+-$ 100 , cal BC 16600-15400), which corresponds to the Lower Dryas, and therefore to the first part of the Middle Magdalenian [9]. It confirms the initial chronological attribution of part of this settlement, based on the study of archaeological remains [15-17]. The number of Middle Magdalenian sites that yield a bone industry has recently increased (3) $[6,10,13,25,26]$. This date also allows us to set the use of foreshafts by some Magdalenian hunters, at least from the start of the Lower Dryas in the Massif Central. If it is possible that such weapons were already used during the Aurignacian period, as an artefact from Castanet indicates, it is still a unique case: the 28 other objects inventoried so far, which are morphologically different from the one at the 'Petits Guinards', have been found in archaeological levels located between the recent part of the Middle Magdalenian and the start of the Upper Magdalenian [4]. Such a radiocarbon date (indicating the start of the Middle Magdalenian) changes our way of considering hunting strategies (and their possible evolution) and the economic and social (?) status of this weapon.

\section{Beyond this item: mobility of human groups and} settlement patterns

The general study of reindeer antlers from the 'Petits Guinards' has shed light on the scarcity of male antlers, as well as on the absence of male antlers whose compact bone tissue width was more than or equal to $10 \mathrm{~mm}$ [6]. So the antler from which the foreshaft is made comes from a reindeer not represented at the site, which means that it was made in another place, before the occupation of the site. This example of spatial fragmentation of the 'chaîne opératoire' does not concern only the bone industry. Indeed, the techno-economic study of the lithic remains proved that the blades made in the 'silex blond' from the Touraine region were not produced on site [19], which means that these blades were introduced on site as unretouched blanks or tools [17]. If such an evidence about a distant origin of the 'silex blond', also demonstrated for some other sites from the Massif Central [16,25], is not really a new information, it is the first time we demonstrate that a worked reindeer antler from the Massif Central was manufactured at a site other than the one where it was found. Are there any further implications regarding our knowledge of the exploitation and mobility patterns of Magdalenian groups in the Massif Central, if we keep in mind that we already know their territory probably spread from the Touraine in the north to the Loire and Allier upper valleys to the south? [14]. None directly, since we do not know of any similar item in the area made in 'silex blond', evidence that would allow us to link the two regions. Indirectly, the discovery of the foreshaft from les Petits Guinards, as part of a large bone industry assemblage, and the fact that it is partially decorated suggest that the status of that bone industry was perhaps more important in the Magdalenian economy of the Massif Central than had been recognized. The fact that this foreshaft was made at another place could be an indication of seasonal production of bone equipment during the cold period, as already proposed for the Upper Palaeolithic of southwestern France $[2,5,13]$. Seasonal data of reindeer antler procurement and exploitation from the 'Petits Guinards' and comparisons with those from northern sites should allow us to test this hypothesis.

\section{Perspectives}

Now that this foreshaft - as well as a second one, reinvested in a chisel - has been identified, it could be appropriate to re-examine other local collections. Indeed, at the scale of the Massif Central and even of the whole country, this kind of artefacts is still exceptional. Does such a scarcity reveal an archaeological reality? Would any broken and/or reinvested bone tool, such as the second one from the 'Petits Guinards', prevent researchers from determining them accurately as a foreshaft? At this stage, we are indeed wondering how this object would have been identified if only the striated bevel or the central part had been discovered. Above all, it is the association groove/bevel that constitutes the ground for such an interpretation. This discovery also leads us to think about settlement patterns, in terms of the status of this site and its place in the annual cycle. On that respect, this research design also includes other sites from the Massif central region as part of an inter-disciplinary archaeological project underway $[6,7,14]$.

\section{Introduction}

Découvert en 1981 lors de travaux de voirie, le gisement archéologique des Petits Guinards, localisé en bord d'Allier sur la commune de Creuzier-le-Vieux (Allier), a été considéré initialement comme un site de plein air. Dans le cadre d'un programme de recherche concernant le Paléolithique supérieur du département de l'Allier, deux nouvelles séries de sondages, menées en 
2002-2003 par deux d'entre nous (LF, LL), ont montré qu'il était en réalité formé de dépôts secondaires issus d'une loupe de glissement et qu'il se situait, à l'origine, à une centaine de mètres en contre-haut, au pied d'un escarpement calcaire. Toutefois, en dehors d'une zone constituée d'un paléochenal, l'organisation interne de la stratigraphie ne semble avoir subi aucun remaniement sédimentaire majeur $[15,16]$. L'échantillon archéologique (125000 vestiges lithiques et osseux) traduit un ensemble ouvert et est donc représentatif, comme le sont généralement les ensembles issus de grottes et abris sous roche $[11,12]$.

Alors que les industries osseuses sont rares dans les assemblages du Paléolithique supérieur régional [6], le gisement des Petits Guinards se singularise par un nombre important (900) de fragments de bois de renne (chute et massacre) et par 74 objets travaillés en matières dures d'origine animale (os, ivoire, bois de cervidés, dent et coquille). Intégrée dès le départ dans le champ de l'analyse archéozoologique, l'étude de cet ensemble s'inscrit dans une problématique de recherche plus large, menée à l'échelle du Paléolithique supérieur du Massif central, qui a pour objectif de replacer ces industries mal connues dans leurs dimensions économique, technique et fonctionnelle. L'analyse de cet assemblage a distingué une pièce exceptionnelle en bois de renne, dont nous pensons qu'il s'agit d'une préhampe, c'est-àdire un élément d'armature composite qui s'intercale entre la hampe d'un projectile et l'extrémité vulnérante (pointe). Elle a été datée dans le cadre d'un programme de datations directes d'objets façonnés en matières dures d'origine animale (Institut de physique des particules de Zürich), mis en place en raison de l'absence d'équivalents régionaux de certaines pièces. C'est cet objet, brisé en cinq fragments et malheureusement incomplet, que nous présentons ici. De par ses caractéristiques volumétriques et fonctionnelles, il s'impose comme une pièce d'exception, porteuse d'informations essentielles relatives aux groupes humains paléolithiques de ce secteur septentrional du Massif central.

\section{Matière, techniques et hypothèses fonctionnelles}

La pièce a été confectionnée dans un bois de renne dont la structure du tissu spongieux, bien visible, est constituée d'alvéoles étroites et serrées. L'épaisseur maximale du tissu compact $(10 \mathrm{~mm})$ indique qu'il s'agissait d'un bois de gros module appartenant à un mâle adulte (Fig. 1). D'une rectitude et d'une régularité complètes sur toute sa longueur $(290 \mathrm{~mm})$, l'objet est affecté d'une fracture en languette à son extrémité la plus large. Il présente une section rectangulaire aux angles légèrement arrondis sur la quasi-totalité de sa longueur. Il s'amincit et s'aplatit progressivement vers l'extrémité la moins large, constituée d'un double biseau de section ovale, strié sur une seule face. À son extrémité fracturée, deux rainures axiales ont été creusées aux dépens des tissus spongieux et compact. Si le façonnage a fait disparaître toute trace de débitage, il ne fait aucun doute que l'extraction de la perche s'est faite par double rainurage longitudinal, technique dont les témoins sont nombreux sur d'autres pièces des Petits Guinards. La pièce a ensuite été raclée, puis polie.

L'objet a donc conservé, en partie ou en totalité, deux aménagements de type fonctionnel. Le premier est constitué d'un long biseau strié, destiné à faciliter son encollage sur un autre élément : la pièce devait être emmanchée de manière fixe à cet endroit, ce qui n'était pas le cas à l'autre extrémité, qui porte une fracture en languette, consécutive à une contrainte par flexion, évoquant l'absence de ligature $[1,24]$. Le second aménagement consiste en deux rainures localisées à l'extrémité fracturée de la pièce. Si leur longueur initiale ne peut être déterminée, leur épaisseur et leur profondeur sont connues. La rainure creusée sur la face de tissu compact est en $《 \mathrm{~V}$ » à fond plat ( $l=4 \mathrm{~mm} ; e=4 \mathrm{~mm})$. Celle aménagée dans la spongiosa est beaucoup plus altérée et largement évasée $(1=5 \mathrm{~mm}$; é $=4 \mathrm{~mm})$. Ces rainures étaient destinées à l'insertion d'autres éléments, sans lesquels l'objet ne pouvait être efficace. Ce type d'aménagement est partiellement visible sur un autre objet des Petits Guinards : un ciseau réalisé à partir d'une pièce recyclée de même calibre, probablement issue de la même catégorie fonctionnelle que celle qui nous occupe dans cet article (Fig. 2). Les deux pièces ne constituent pas un seul et même objet.

Nous sommes donc en présence d'un élément constitutif d'un objet technique composite emmanché de manière fixe et dont la structure générale évoque la sphère des projectiles. L'emmanchement de cet élément sur une hampe en bois végétal reste probable. L'interprétation comme un élément de la partie distale d'un propulseur, encollée sur un manche en bois végétal et dont le crochet serait rapporté, semble devoir être éliminée. Elle s'accorde mal avec ce que l'on connaît de l'emmanchement des parties proximales biseautées des propulseurs paléolithiques, dans la mesure où le biseau, simple ou double, est toujours parallèle à l'axe du crochet, alors qu'il serait perpendiculaire dans le cas de la pièce des Petits Guinards [23]. En outre, dans une telle hypothèse d'utilisation, on conçoit mal que l'objet, dont la résilience est considérablement amoindrie par la présence des profondes rainures axiales, supporte les for- 

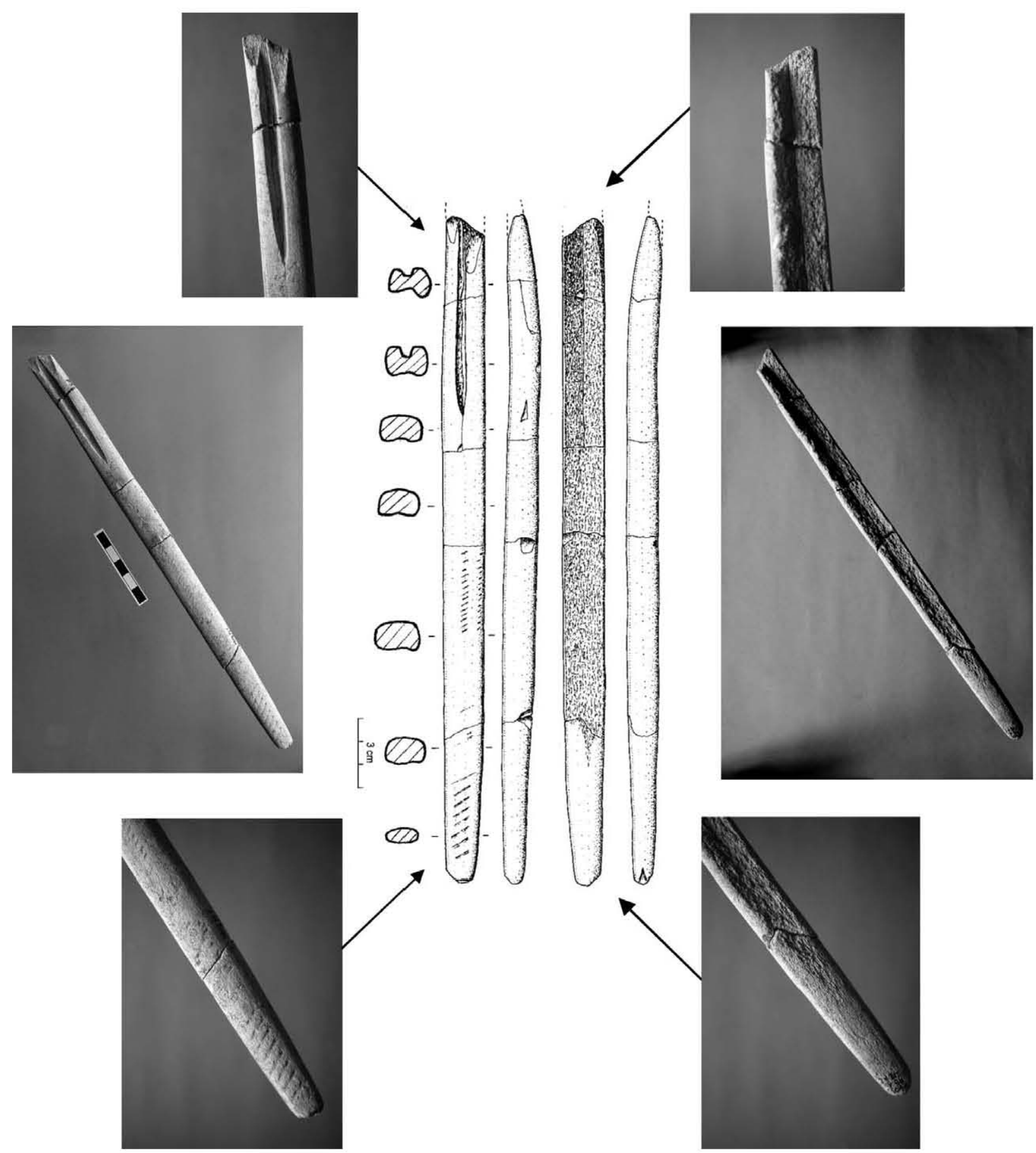

Fig. 1. Les Petits Guinards à Creuzier-le-Vieux (Allier). Pièce en bois de renne interprétée comme une préhampe de projectile. Fig. 1. Petits Guinards (Creuzier-le-Vieux, Allier, France). Worked Reindeer antler interpreted as a foreshaft of projectile.

ces exercées perpendiculairement à sa plus grande longueur et à sa plus grande épaisseur sans se fracturer.

Une des réponses se trouve dans la destination fonctionnelle des rainures, dont la largeur et la profondeur sont largement supérieures à celles de la plupart des têtes de projectiles rainurées étudiées à partir d'autres séries archéologiques [18]. L'hypothèse d'une fixation, à l'aide d'un adhésif, de pièces lithiques telles que des lamelles à dos se heurte à deux problèmes. Dans un premier temps, si l'on confronte le calibre général des 
lamelles à dos des Petits Guinards à la largeur et à la profondeur des rainures de la préhampe, ces dernières sont trop profondes et trop larges pour laisser dépasser le tranchant de la plupart de ces éléments lithiques, qui deviendraient dès lors inefficaces. L'attribution chronotypologique (Épipaléolithique ?) des seules pièces à dos qui pourraient effectivement s'insérer dans les rainures ne concorde pas avec celle donnée par la datation directe de la préhampe (cf. ci-dessous). Mais, dans tous les cas, la section rectangulaire de l'objet semble impropre à une utilisation comme tête de projectile et la partie fracturée n'a certainement pas été appointée.

La morphologie de la pièce ainsi que l'incompatibilité des rainures avec les pièces à dos nous amènent donc à retenir l'hypothèse d'une préhampe, dont on connaît quelques exemplaires au Paléolithique supérieur [4,20-22]. Il est en fait fort probable que les rainures ont servi à insérer, soit une pointe vulnérante, soit

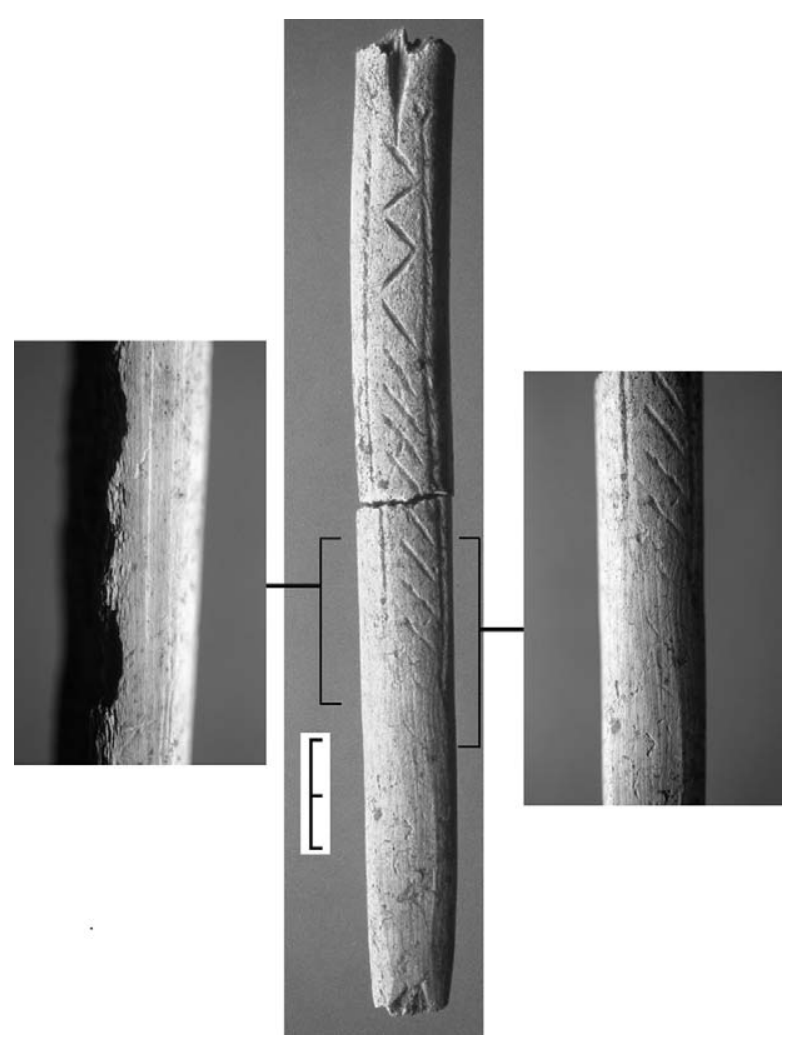

Fig. 2. Les Petits Guinards à Creuzier-le-Vieux (Allier). Autre pièce en bois de renne interprétée comme une préhampe de projectile, recyclée en ciseau. Détails de l'extraction (à gauche) et les traces de raclage postérieures au décor (à droite) (échelle : $2 \mathrm{~cm}$ ).

Fig. 2. Petits Guinards (Creuzier-le-Vieux, Allier, France). Another worked reindeer antler interpreted as a foreshaft projectile, reprocessed as a chisel. Detail of extraction (left) and traces of scraping (right) (scale: $2 \mathrm{~cm}$ ). une autre pièce intermédiaire. Mais on voit mal quel élément unique aurait pu s'insérer : des pointes, ou d'autres éléments en matières osseuses à base fourchue, seraient inadéquates car, d'une part, la fourche de l'objet inséré aurait dû être beaucoup plus longue que toutes celles archéologiquement attestées [8,21,22] et, d'autre, part, ce type de pointes n'existe pas aux Petits Guinards. Reste l'hypothèse, non vérifiable, d'une pointe ou d'une autre préhampe fourchue en bois végétal.

L'analyse contextuelle du matériel permet d'envisager, à titre d'hypothèse, l'insertion de pointes en matières osseuses, identiques à celles découvertes dans le gisement. Rectilignes, de section triangulaire, ces pointes - ou plutôt bi-pointes - pouvaient être armées ou non de barbelures lithiques. Le maintien du montage pouvait être assuré au moyen d'un adhésif ou d'une ligature (Fig. 3). Dans ce dernier cas, la ligature n'était

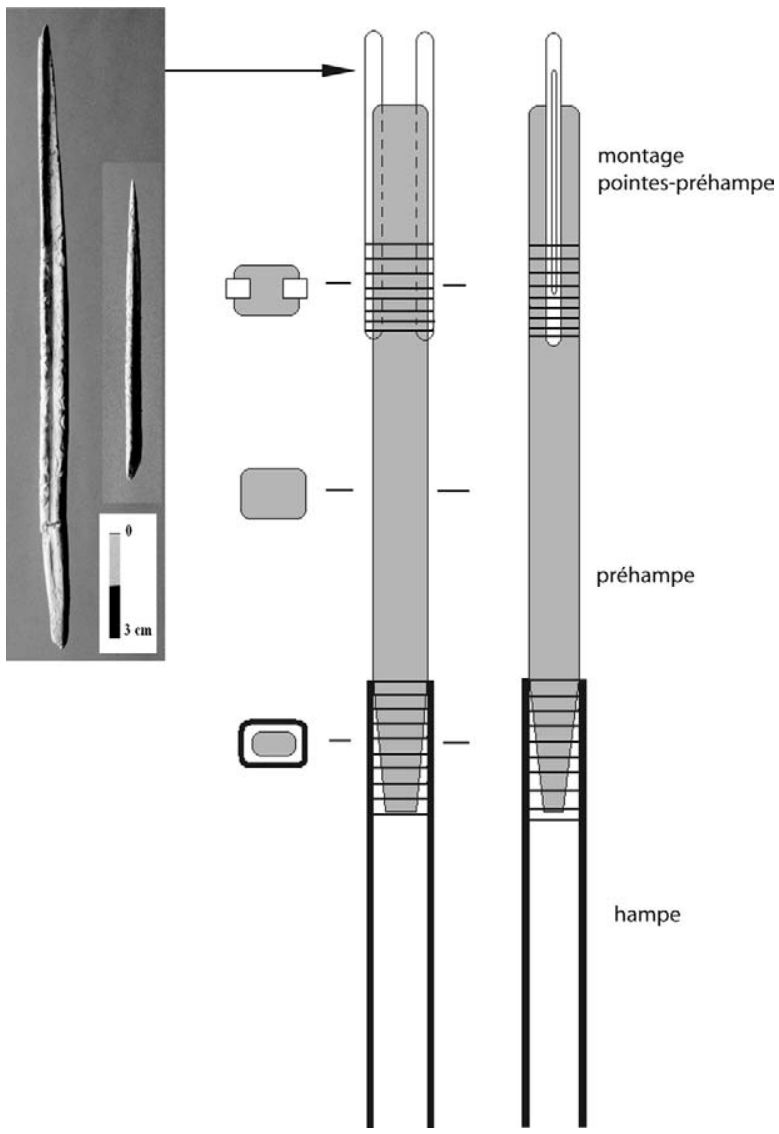

Fig. 3. Les Petits Guinards à Creuzier-le-Vieux (Allier). Hypothèse de montage de pointes en matières osseuses dans les rainures de la préhampe (échelle : $2 \mathrm{~cm}$ ).

Fig. 3. Petits Guinards (Creuzier-le-Vieux, Allier, France). Hypothetical setting of worked bone points in the grooves of the foreshaft projectile (scale: $2 \mathrm{~cm}$ ). 
pas placée au niveau de l'extrémité distale actuelle, mais nécessairement plus bas, sur la préhampe, comme l'atteste la fracture en languette, ce qui a permis par ailleurs la propagation de l'onde de fracture. Finalement, le poids conséquent du montage évoque davantage un lanceur de type propulseur ou une arme d'estoc qu'un arc [3].

Il s'agit d'une arme exceptionnelle, connue uniquement dans sept sites français (abri du Colombier, Ardèche ; Tuc d'Audoubert, Ariège ; abri Castanet, Longueroche et la Madeleine, Dordogne ; Isturitz, PyrénéesAtlantiques ; abri de Fontalès, Tarn-et-Garonne) [4]. Sa présence (sous la forme de deux objets fragmentés, cf. ci-dessus) dans une région où l'industrie osseuse est rarissime soulève certaines questions.

Quant à sa présence sur le site, elle peut s'expliquer par l'éventuelle nécessité de récupérer la hampe en bois végétal (rectiligne et de grandes dimensions), liée ou non à la rareté en bois d'origine végétale au Dryas ancien. Cette activité de démontage du système de fixation, qui requiert une source de chaleur et un minimum de temps et de confort, s'exerçait peut-être plus à l'intérieur d'une habitation que sur le lieu de chasse ou de pêche. La pièce a également pu être rapportée pour être recyclée, notamment en pièce intermédiaire de type ciseau, comme l'a été l'autre fragment de préhampe mis au jour.

\section{Datation directe de la préhampe : implications}

La datation SMA ${ }^{14} \mathrm{C}$ obtenue (ETH-28777, 14970 \pm 100 , cal BC 16600-15400) correspond au Dryas ancien et renvoie à la phase ancienne du Magdalénien moyen [9]. Elle confirme l'attribution initiale d'une partie des occupations du gisement, réalisée à la suite du diagnostic du matériel archéologique [15-17]. Elle vient enrichir le corpus des dates les plus récentes qui ont permis de réattribuer au Magdalénien moyen les gisements voisins du Puy-de-Dôme (Lépétade-Chabasse, Auzary-Thônes et le Bay). Elle porte désormais à trois (Auzary-Thônes, le Bay et les Petits Guinards) le nombre de sites où l'industrie osseuse de cette phase du Magdalénien est sûrement représentée [6,10,13,25,26].

Cette datation permet également de situer l'utilisation de préhampes dans la panoplie de certains chasseurs magdaléniens, au moins à partir du début du Dryas ancien pour le Massif central. Si l'exemplaire de l'abri Castanet laisse envisager leur emploi à l'Aurignacien, il constitue un cas isolé : les 28 autres pièces connues, morphologiquement différentes de celle des Petits Guinards, sont attribuées, par leur contexte archéologique, à la transition entre la phase récente du
Magdalénien moyen et le tout début du Magdalénien supérieur [4]. Une telle datation renouvelle donc la problématique des stratégies de chasse et de leur éventuelle évolution.

\section{Au-delà de l'objet : circulation des groupes humains et territoires}

L'étude globale des bois de renne des Petits Guinards (chute, massacre, fragments, déchets de débitage) a montré la rareté des bois de mâles et l'absence de bois de mâle adulte dont l'épaisseur de tissu compact était supérieure ou égale à $10 \mathrm{~mm}$ [6]. Le bois dans lequel la préhampe a été confectionnée provient donc d'un individu non représenté dans le gisement, ce qui atteste sa fabrication antérieure et hors du site.

Cet exemple de fragmentation spatiale de la chaîne opératoire ne concerne pas seulement l'industrie osseuse. En effet, l'étude techno-économique du matériel lithique a démontré l'absence de production, in situ, des lames en silex blond de Touraine, ce qui implique leur introduction dans le gisement à l'état de produits laminaires ou d'outils [17]. Si cette donnée relative à la provenance lointaine de produits laminaires en silex blond, déjà démontrée sur de nombreux gisements du Massif central [19], ne constitue pas une nouveauté, c'est la première fois qu'une production hors site d'un objet en bois de renne est mise en évidence dans cette vaste région. Cela apporte-t-il des éléments nouveaux pour la connaissance des modalités d'exploitation du territoire des Magdaléniens du Massif central, dont il est possible qu'il s'étendît de la Touraine, au nord, jusqu'aux hautes vallées de la Loire et de l'Allier, au sud ? [14]. Pas directement, puisque aucun objet comparable n'est connu dans la région d'approvisionnement en silex blond, qui permettrait d'établir une liaison supplémentaire entre les deux régions. Indirectement, la découverte d'un tel objet au sein d'un assemblage important en matière dure d'origine animale, de surcroît en partie décoré, suggère une place peut-être plus importante qu'on ne l'a dit jusque là de l'industrie osseuse dans l'économie des Magdaléniens du Massif central [6]. Le fait que cette préhampe ait été fabriquée ailleurs, comme une partie de l'industrie osseuse, est peut-être un indice de fabrication saisonnière de l'équipement en bois de renne, argumentée ou envisagée à titre hypothétique, en saison froide, pour le Paléolithique supérieur du Sud-Ouest français $[2,5,13]$. La confrontation des données relatives à la saison d'acquisition et d'exploitation des bois de renne des Petits Guinards à celles de sites du Nord devrait permettre de traiter une partie de la question. 


\section{Perspectives}

L'identification de cette préhampe - et de son équivalent recyclé en ciseau - ouvre le champ au réexamen de certaines collections régionales. En effet, à l'échelle du Massif central et même de la France, la présence d'un tel objet demeure exceptionnelle. Cette rareté correspond-elle à une réalité archéologique, ou le caractère fragmentaire et la réutilisation de cette pièce ont-t-ils empêché son identification ? En effet, on peut se demander dans quelle catégorie ce fragment aurait été classé si seul le biseau strié ou la partie mésiale avaient été découverts : c'est avant tout l'association rainures/ biseau qui constitue l'argument d'une interprétation comme préhampe.

Cette découverte pose également la question du statut des Petits Guinards et celle de sa place dans le cycle annuel en termes de saison et d'activité, question qui constitue la thématique dominante dans l'étude interdisciplinaire en cours $[6,7,14]$.

\section{Remerciements}

Nous remercions P. Cattelain et C. Bellier, musée du Malgré Tout (Treignes, Belgique), ainsi que J.-M. Pétillon, pour nous avoir fait part de leurs hypothèses interprétatives de la pièce décrite dans cet article. Nos remerciements s'adressent également à J. Roethlisberger et M. Juillard, infographistes et photographes au Laténium, ainsi qu'à S. Renault, MMSH-CNRSUMR 6636, pour la réalisation de certaines des figures du manuscrit, et enfin à C. Delage, pour la correction de la version anglaise.

\section{Références}

[1] J. Allain, A. Rigaud, Décor et fonction. Quelques exemples tirés du Magdalénien, Anthropol. 90 (4) (1986) 713-738.

[2] A. Averbouh, R. Bégouën, J. Clottes, Technique et économie du travail du bois de cervidé chez les Magdaléniens d'Enlène (Montesquieu-Avantès, Ariège) ; vers l'identification d'un cycle saisonnier de production?, in: M. Julien, A. Averbouh, D. Ramseyer, C. Bellier, D. Buisson, P. Cattelain, M. PatouMathis, N. Provenzano (Eds.), Préhistoire d'os. Recueil d'études sur l'industrie osseuse préhistorique, Publications de l'université de Provence, Aix-en-Provence, 1999, pp. 289-318.

[3] P. Carrère, S. Lepetz, Étude de la dynamique des pointes de projectiles : élaboration d'une méthode, mémoire de maitrise, université Paris-1-Panthéon-Sorbonne, 1988.

[4] P. Cattelain, avec la collaboration de G. Onoratini, Fiche éléments intermédiaires de hampes de projectiles, in: J. Allain, A. Averbouh, H. Barge-Mahieu, C. Beldiman, D. Buisson, H. Camps-Fabrer, P. Cattelain, S.-Y. Choi, J.-G. Nandris, M. Patou-Mathis, A. Peltier, N. Provenzano, D. Ramseyer (Eds.), Éléments récepteurs, fiches typologiques de l'industrie osseuse préhistorique, Union internationale des sciences préhistoriques et protohistoriques, Commission de nomenclature sur l'industrie de l'os préhistorique, 6, Cedarc, Treignes, 1993, pp. 15-22.

[5] C. Chauchat, D. Bonnissent, F.-X. Chauvière, M. Dachary, C. Fritz, P. Fosse, A. Esatham, H. Martin, O. Le Gall, D. Gambier, A. Roussot, M. Fontugne, C. Hatte, L'habitat magdalénien du Bourouilla à Arancou (Pyrénées-Atlantiques, France), Gallia Préhistoire 41 (1999) 1-151.

[6] F.-X. Chauvière, L. Fontana, Exploitation du monde animal du Châtelperronien au Mésolithique dans le Massif central : études anciennes, approches récentes, in: J.-P. Raynal (Ed.), 19042004. Un siècle de préhistoire et de protohistoire dans le Massif central : bilans et perspectives, CDDP de Haute-Loire et musée Crozatier, Le Puy-en-Velay, 22-24 octobre 2004 (à paraître).

[7] F.-X. Chauvière, L. Fontana, Modalités d'exploitation des rennes du Blot (Haute-Loire) : entre subsistance, technique et symbolique, in: V. Dujardin (Ed.), Industrie osseuse et parures du Solutréen au Magdalénien en Europe, Table ronde sur le Paléolithique supérieur récent, Angoulême 28-30 mars 2003, 2005, pp. 137-147.

[8] H. Delporte, L. Mons, Fiche « sagaie à base fourchue », in: H. Delporte, J. Hahn, L. Mons, G. Pinçon, D. De Sonneville-Bordes (Eds.), Sagaies, Fiches typologiques de l'industrie osseuse préhistorique, Union internationale des sciences préhistoriques et protohistoriques, Commission de nomenclature sur l'industrie de l'os préhistorique, 1, Publications de l'université de Provence, Aix-en-Provence, 1988 (11 p).

[9] F. Djindjian, J. Koslowski, M. Otte, Le Paléolithique supérieur en Europe, Armand Colin, Paris, 1999.

[10] L. Fontana, Stratégies de subsistance au Badegoulien et au Magdalénien en Auvergne : nouvelles données, in: G. Pion (Ed.), Le Paléolithique supérieur récent : nouvelles données sur le peuplement et l'environnement, Actes de la table ronde de Chambéry, 12-13 mars 1999, Société préhistorique française, mémoire XXVIII, Paris, 2000, pp. 59-65.

[11] L. Fontana, La chasse au Renne au Paléolithique supérieur : nouvelles voies de recherche, Paléo 12 (2000) 141-164.

[12] L. Fontana, Territoires, mobilité et échanges au Magdalénien dans l'Aude et le Massif central (France) : approche comparative, modélisation et perspectives, in: Territoires, déplacements, mobilité, échanges. Actes du 126e congrès, CTHS, Toulouse, 2005, pp. 355-370.

[13] L. Fontana, Nouvelles datations radiocarbone pour le Paléolithique supérieur du Massif central (France) : enjeux et perspectives, inédit.

[14] L. Fontana, F.-X. Chauvière, L. Lang, Comment appréhender les modes d'acquisition et d'exploitation des sociétés du Paléolithique supérieur? Méthodologie et étude de cas, in: S.A. de Beaune (Ed.), Restituer la vie quotidienne au Paléolithique supérieur, colloque international, Lyon, 16-18 mars, 2005 (à paraître).

[15] L. Fontana, L. Lang, F.-X. Chauvière, M. Jeannet, L. Magoga, Nouveau sondage sur le site paléolithique des Petits Guinards à Creuzier-le-Vieux (Allier, France) : des données inattendues, Bull. Soc. Préhist. Fr. 100 (3) (2003) 591-596.

[16] L. Fontana, L. Lang, F.-X. Chauvière, M. Jeannet, L. Magoga, Le Paléolithique supérieur récent du Nord du Massif central : des données inattendues sur le site des Petits Guinards à Creuzier-le-Vieux (Allier, France), Préhist. Sud-Ouest 10 (1) (2003) 77-93.

[17] L. Fontana, L. Lang, F.-X. Chauvière, M. Jeannet, L. Magoga, C. Mourer-Chauviré, Les Petits Guinards, commune de Creuzier-le-Vieux (Allier). Campagnes du 14/07/03 au 10/08/03, 
rapport de sondage et prospection thématique, Aix-en-Provence, 2003.

[18] C. Houmard, Réflexion sur les tes têtes de projectiles rainurées d'après l'étude du site de la Garenne (Indre), Préhist. Anthropol. Méditerr. 12 (2003) 165-172.

[19] A. Masson, Pétroarchéologie des roches siliceuses. Intérêt en préhistoire, thèse de $3^{\mathrm{e}}$ cycle, université Lyon-1, 1981.

[20] J.-M. Pétillon, Typologie et utilisation : l'exemple des pointes à base fourchue magdaléniennes, in: M. Patou-Mathis, P. Cattelain, D. Ramseyer (Eds.), L'industrie osseuse pré- et protohistorique en Europe. Approches techniques et fonctionnelles, Actes du colloque 1.6, XIV ${ }^{\mathrm{e}}$ Congrès de l'UISPP, Liège, Belgique, 2-8 septembre 2001, XXVI, 2003, pp. 53-62.

[21] J.-M. Pétillon, Des Magdaléniens en armes. Technologie des armatures de projectiles en bois de cervidé du Magdalénien supérieur de la grotte d'Isturitz (Pyrénées-Atlantiques), thèse, université Paris-1-Panthéon-Sorbonne, 2004.

[22] J.-M. Pétillon, P. Cattelain, Nouvel examen de l'armature composite magdalénienne du Tuc d'Audoubert (Montesquieu-Avantès, Ariège), Bull. Soc. Préhist. Fr. 101 (4) (2004) 45-53.
[23] U. Stodiek, À propos de l'emmanchement des propulseurs au Paléolithique supérieur, in: Le peuplement magdalénien. Paléogéographie physique et humaine, Colloque de Chancelade, octobre 1988, CTHS, Paris, 1992, pp. 317-331.

[24] U. Stodiek, Preliminary Results of an experimental investigation of Magdalenian antler points, in: C. Bellier, P. Cattelain, M. Otte (Eds.), La chasse dans la préhistoire/Hunting in Prehistory, Actes du Colloque international de Treignes, 3-7 octobre 1990, Éditions du Cedarc, Anthropologie et préhistoire, Artefacts 8, ERAUL 51, Treignes, 2000, pp. 70-78.

[25] F. Surmely, Le peuplement magdalénien de l'Auvergne, in: G. Pion (Ed.), Le Paléolithique supérieur récent : nouvelles données sur le peuplement et l'environnement, Actes de la table ronde de Chambéry, 12-13 mars 1999, Société préhistorique française, mémoire XXVIII, Paris, 2000, pp. 165-175.

[26] F. Surmely, La datation du Magdalénien en Auvergne, in: J.-N. Barrandon, P. Guibert, V. Michel (Eds.), Datation, XXI ${ }^{\mathrm{e}}$ Rencontres internationales d'archéologie et d'histoire d'Antibes, Actes des rencontres, 19-21 octobre 2001, Éditions ADPCA, Antibes, 2001, pp. 349-359. 
